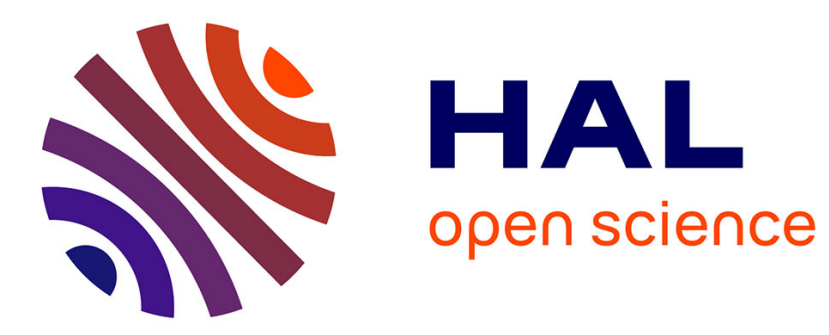

\title{
Subset Simulation and Line Sampling for Advanced Monte Carlo Reliability Analysis
}

\author{
Enrico Zio, Nicola Pedroni
}

\section{To cite this version:}

Enrico Zio, Nicola Pedroni. Subset Simulation and Line Sampling for Advanced Monte Carlo Reliability Analysis. Proceedings of the European Safety and RELiability (ESREL) 2009 Conference, 2009, pp.687-694. hal-00721047

\section{HAL Id: hal-00721047 \\ https://hal.science/hal-00721047}

Submitted on 26 Jul 2012

HAL is a multi-disciplinary open access archive for the deposit and dissemination of scientific research documents, whether they are published or not. The documents may come from teaching and research institutions in France or abroad, or from public or private research centers.
L'archive ouverte pluridisciplinaire HAL, est destinée au dépôt et à la diffusion de documents scientifiques de niveau recherche, publiés ou non, émanant des établissements d'enseignement et de recherche français ou étrangers, des laboratoires publics ou privés. 


\title{
Subset Simulation and Line Sampling for Advanced Monte Carlo Reliability Analysis
}

\author{
E. Zio \& N. Pedroni \\ Energy Department, Politecnico di Milano, Milano, Italy
}

\begin{abstract}
In this paper, the recently developed Subset Simulation (SS) and Line Sampling (LS) techniques are considered for improving the efficiency of Monte Carlo Simulation (MCS) in the estimation of system failure probability. The SS method is founded on the idea that a small failure probability can be expressed as a product of larger conditional probabilities of some intermediate events: with a proper choice of the intermediate events, the conditional probabilities can be made sufficiently large to allow accurate estimation with a small number of samples. The LS method employs lines instead of random points in order to probe the failure domain of interest. An "important direction" is determined, which points towards the failure domain of interest; the high-dimensional reliability problem is then reduced to a number of conditional one-dimensional problems which are solved along the "important direction".
\end{abstract}

The two methods are applied on a structural reliability model of literature. The efficiency of the proposed techniques is evaluated in comparison to the commonly adopted standard MCS.

\section{INTRODUCTION}

In the performance-based design and operation of modern engineered systems, the accurate assessment of reliability is of paramount importance, particularly for civil, nuclear, aerospace and chemical systems and plants which are safety-critical and must be designed and operated within a risk-informed approach (Patalano et al. 2008).

The reliability assessment requires the realistic modelling of the structural/mechanical components of the system and the characterization of their material constitutive behaviour, loading conditions and mechanisms of deterioration and failure that are anticipated to occur during the working life of the system (Schueller \& Pradlwarter 2007).

In practice, not all the characteristics of the system under analysis can be fully captured in the model. This is due to: i) the intrinsically random nature of several of the phenomena occurring during the system life; ii) the incomplete knowledge about some of these phenomena. Thus, uncertainty is always present in the hypotheses underpinning the model (model uncertainty) and in the values of its parameters (parameter uncertainty); this leads to uncertainty in the model output, which must be quantified for a realistic assessment of the system (Nutt \& Wallis 2004).

In mathematical terms, the probability of system failure can be expressed as a multi-dimensional integral of the form

$$
P(F)=P(\underline{x} \in F)=\int I_{F}(\underline{x}) q(\underline{x}) d \underline{x}
$$

where $\underline{x}=\left\{x_{1}, x_{2}, \ldots, x_{j}, \ldots, x_{n}\right\} \in \mathfrak{R}^{n}$ is the vector of the random states of the components, i.e. the random configuration of the system, with multidimensional probability density function (PDF) $q: \mathfrak{R}^{n} \rightarrow[0, \infty)$, $F \subset \mathfrak{R}^{n}$ is the failure region and $I_{F}: \mathfrak{R}^{n} \rightarrow\{0,1\}$ is an indicator function such that $I_{F}(\underline{x})=1$, if $\underline{x} \in F$ and $I_{F}(\underline{x})=0$, otherwise. The failure domain $F$ is commonly defined by a so-called Performance Function (PF) or Limit State Function (LSF) $g_{x}(\underline{x})$ which is lower than or equal to zero if $\underline{x} \in F$ and greater than zero, otherwise.

In practical cases, the multi-dimensional integral (1) can not be easily evaluated by analytical methods nor by numerical schemes. On the other hand, Monte Carlo Simulation (MCS) offers an effective means for estimating the integral, because the method does not suffer from the complexity and dimension of the domain of integration, albeit it implies the nontrivial task of sampling from the multidimensional PDF. The MCS solution to (1) entails that a large number of samples of the values of the uncertain parameters $\underline{x}$ be drawn from $q(\underline{x})$ and that these be used to compute an unbiased and consistent estimate of the system failure probability as the fraction of the number of samples that lead to failure. However, a large number of samples (inversely proportional to the failure probability) is necessary to achieve an acceptable estimation accuracy: in terms of the integral 
in (1) this can be seen as due to the high dimensionality $n$ of the problem and the large dimension of the relative sample space compared to the failure region of interest (Schueller 2007). This calls for new simulation techniques for performing robust estimations with a limited number of input samples (and associated low computational time).

In this respect, effective approaches are offered by Subset Simulation (SS) (Au and Beck 2001, Au \& Beck 2003) and Line Sampling (LS) (Koutsourelakis et al. 2004, Pradlwarter et al. 2005).

In the SS method, the failure probability is expressed as a product of conditional failure probabilities of some chosen intermediate events, whose evaluation is obtained by simulation of more frequent events. The evaluation of small failure probabilities in the original probability space is thus tackled by a sequence of simulations of more frequent events in the conditional probability spaces. The necessary conditional samples are generated through successive Markov Chain Monte Carlo (MCMC) simulations (Metropolis et al. 1953), gradually populating the intermediate conditional regions until the final target failure region is reached.

In the LS method, lines, instead of random points, are used to probe the failure domain of the highdimensional problem under analysis (Pradlwarter et al. 2005). An "important direction" is optimally determined to point towards the failure domain of interest and a number of conditional, one-dimensional problems are solved along such direction, in place of the high-dimensional problem (Pradlwarter et al. 2005). The approach has been shown to perform always better than standard MCS; furthermore, if the boundaries of the failure domain of interest are not too rough (i.e., almost linear) and the "important direction" is almost perpendicular to them, the variance of the failure probability estimator could be ideally reduced to zero (Koutsourelakis et al. 2004).

In this paper, SS and LS schemes are developed for application to a structural reliability model of literature, i.e., the cracked plate model (Ardillon \& Venturini 1995). The problem is rather challenging as it entails estimating failure probabilities of the order of $10^{-7}$.

The benefits gained by the use of SS and LS are demonstrated by comparison with respect to standard MCS.

The remainder of the paper is organized as follows. General presentations of the SS and LS schemes implemented for this study are given in Sections 2 and 3 , respectively. In Section 4, the results of the application of SS and LS to the cracked plate model are reported. Finally, some conclusions are proposed in the last Section.

\section{THE SUBSET SIMULATION METHOD}

\subsection{The basic principles}

For a given target failure event $F$ of interest, let $F_{1}$ $\supset F_{2} \supset \ldots \supset F_{m}$ be a sequence of intermediate failure events, so that $F_{k}=\cap_{i=1}^{k} F_{i}, k=1,2, \ldots, m$. By sequentially conditioning on the event $F_{i}$, the failure probability $P(F)$ can be written as

$$
P(F)=P\left(F_{m}\right)=P\left(F_{1}\right) \prod_{i=1}^{m-1} P\left(F_{i+1} \mid F_{i}\right)
$$

Notice that even if $P(F)$ is small, the conditional probabilities involved in (2) can be made sufficiently large by appropriately choosing $m$ and the intermediate failure events $\left\{F_{i}, i=1,2, \ldots, m-1\right\}$.

The original idea of $\mathrm{SS}$ is to estimate the failure probability $P(F)$ by estimating $P\left(F_{1}\right)$ and $\left\{P\left(F_{i+1} \mid F_{i}\right)\right.$ : $i=1,2, \ldots, m-1\}$. Considering for example $P(F) \approx$ $10^{-5}$ and choosing $m=5$ intermediate failure events such that $P\left(F_{1}\right)$ and $\left\{P\left(F_{i+1} \mid F_{i}\right): i=1,2,3,4\right\} \approx 0.1$, the conditional probabilities can be evaluated efficiently by simulation of the relatively frequent failure events (Au \& Beck 2001).

Standard MCS can be used to estimate $P\left(F_{1}\right)$. On the contrary, computing the conditional failure probabilities in (2) by MCS entails the non-trivial task of sampling from the conditional distributions of $\underline{x}$ given that it lies in $F_{i}, i=1,2, \ldots, m-1$, i.e. from $q\left(\underline{x} \mid F_{i}\right)=q(\underline{x}) I_{F i}(\underline{x}) / P(F)$. In this regard, Markov Chain Monte Carlo (MCMC) simulation provides a powerful method for generating samples conditional on the failure region $F_{i}, i=1,2, \ldots, m-1$ (Au \& Beck 2003). The related algorithm is not reported here for brevity; the interested reader may refer to (Au \& Beck 2003) for details.

\subsection{The algorithm}

In the actual SS implementation, with no loss of generality it is assumed that the failure event of interest can be defined in terms of the value of a critical response variable $Y$ of the system under analysis (e.g., its output performance) being lower than a specified threshold level $y$, i.e., $F=\{Y<y\}$. The sequence of intermediate failure events $\left\{F_{i}: i=1,2, \ldots\right.$, $m\}$ can then be correspondingly defined as $F_{i}=\{Y<$ $\left.y_{i}\right\}, i=1,2, \ldots, m$, where $y_{1}>y_{2}>\ldots>y_{i}>\ldots>y_{m}=$ $y>0$ is a decreasing sequence of intermediate threshold values (Au \& Beck 2001). Notice that since these intermediate threshold values (i.e., failure regions) are introduced purely for computational reasons in SS, they may not have a strict physical interpretation and may not be connected to known degradation processes.

The choice of the sequence $\left\{y_{i}: i=1,2, \ldots, m\right\}$ affects the values of the conditional probabilities $\left\{P\left(F_{i+1} \mid F_{i}\right): i=1,2, \ldots, m-1\right\}$ in (2) and hence the efficiency of the SS procedure. In particular, choos- 
ing the sequence $\left\{y_{i}: i=1,2, \ldots, m\right\}$ arbitrarily a priori makes it difficult to control the values of the conditional probabilities $\left\{P\left(F_{i+1} \mid F_{i}\right): i=1,2, \ldots, m\right.$ $1\}$ in the application to real systems. For this reason, in this work, the intermediate threshold values are chosen adaptively in such a way that the estimated conditional failure probabilities are equal to a fixed value $p_{0}$ : the value $p_{0}$ is chosen to be 0.1 which is empirically found to yield good efficiency ( $\mathrm{Au} \&$ Beck 2001).

The SS algorithm proceeds as follows (Figure 1). First, $N$ vectors $\left\{\underline{x}_{0}{ }^{k}: k=1,2, \ldots, N\right\}$ are sampled by standard MCS, i.e., from the original probability density function $q(\cdot)$. The subscript ' 0 ' denotes the fact that these samples correspond to 'Conditional Level 0 '. The corresponding values of the response variable $\left\{Y\left(\underline{x}_{0}{ }^{k}\right): k=1,2, \ldots, N\right\}$ are then computed (Figure 1a) and the first intermediate threshold value $y_{1}$ is chosen as the $\left(1-p_{0}\right) N^{\text {th }}$ value in the decreasing list of values $\left\{Y\left(\underline{x}_{0}{ }^{k}\right): k=1,2, \ldots, N\right\}$. By so doing, the sample estimate of $P\left(F_{1}\right)=P\left(Y<y_{1}\right)$ is equal to $p_{0}$ (note that it has been implicitly assumed that $p_{0} N$ is an integer value) (Figure 1b). With this choice of $y_{1}$, there are now $p_{0} N$ samples among $\left\{\underline{x}_{0}{ }^{k}: k=1,2\right.$, $\ldots, N\}$ whose response $Y$ lies in $F_{1}=\left\{Y<y_{1}\right\}$. These samples are at 'Conditional level 1' and distributed as $q\left(\cdot \mid F_{1}\right)$. Starting from each one of these samples, MCMC simulation is used to generate $\left(1-p_{0}\right) N$ additional conditional samples distributed as $q\left(\cdot \mid F_{1}\right)$, so that there are a total of $N$ conditional samples $\left\{\underline{x}_{1}{ }^{k}: k\right.$ $=1,2, \ldots, N\} \in F_{1}$, at 'Conditional level 1' (Figure $1 \mathrm{c})$. Then, the intermediate threshold value $y_{2}$ is chosen as the $\left(1-p_{0}\right) N^{\text {th }}$ value in the descending list of $\left\{Y\left(\underline{x}_{1}{ }^{k}\right): k=1,2, \ldots, N\right\}$ to define $F_{2}=\left\{Y<y_{2}\right\}$ so that, again, the sample estimate of $P\left(F_{2} \mid F_{1}\right)=P(Y<$ $\left.y_{2} \mid Y<y_{1}\right)$ is equal to $p_{0}$ (Figure 1d). The $p_{0} N$ samples lying in $F_{2}$ are conditional values from $q\left(\cdot \mid F_{2}\right)$ and function as 'seeds' for sampling $\left(1-p_{0}\right) N$ additional conditional samples distributed as $q\left(\cdot \mid F_{2}\right)$, making up a total of $N$ conditional samples $\left\{\underline{x}_{2}: k=1,2, \ldots, N\right\}$ at 'Conditional level 2'. This procedure is repeated for the remaining conditional levels until the samples at 'Conditional level $(m-1)$ ' are generated to yield $y_{m}<y$ as the $\left(1-p_{0}\right) N^{\text {th }}$ value in the descending list of $\left\{Y\left(x_{m-1}\right): k=1,2, \ldots, N\right\}$, so that there are enough samples for estimating $P(Y<y)$ (Au \& Beck 2003).

\section{THE LINE SAMPLING METHOD}

Line Sampling (LS) was also originally developed for the reliability analysis of complex structural systems with small failure probabilities (Koutsourelakis et al. 2004). The underlying idea is to employ lines instead of random points in order to probe the failure domain of the high-dimensional system under analysis (Pradlwarter et al. 2005).

In extreme synthesis, the problem of computing the multidimensional failure probability integral (1) in the original "physical" space is transformed into the so-called "standard normal space", where each
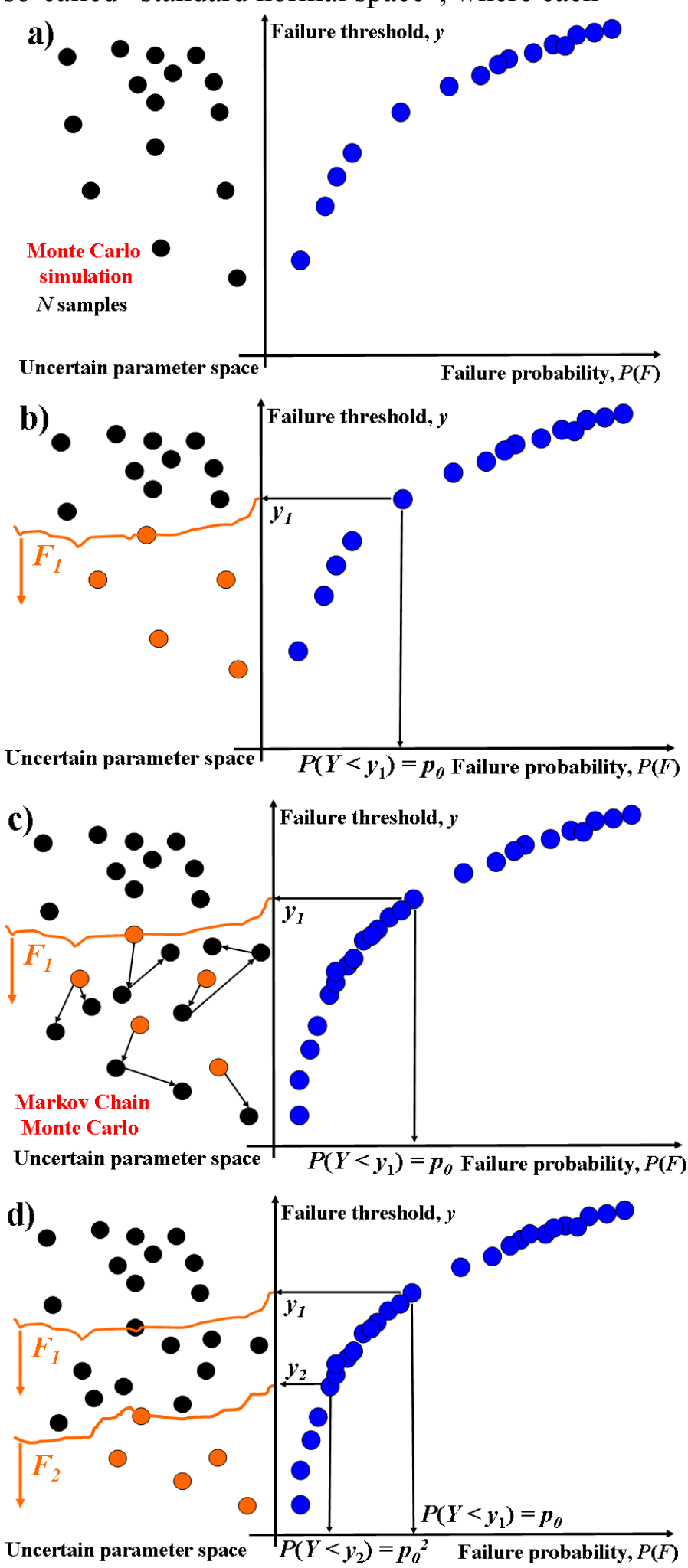

Figure 1. Illustration of the SS procedure: a) Conditional level 0: Standard Monte Carlo simulation; b) Conditional level 0: adaptive selection of $y_{1}$; c) Conditional level 1: Markov Chain Monte Carlo simulation; d) Conditional level 1: adaptive selection of $y_{2}$.

random variable is represented by an independent central unit Gaussian distribution. In this space, a unit vector $\underline{\alpha}$ (hereafter also called "important unit vector" or "important direction") is determined, pointing towards the failure domain $F$ of interest (for illustration purposes, two different failure domains, 
$F^{1}$ and $F^{2}$, are visually represented in Figure 2, left and right, respectively, in a two-dimensional uncertain parameter space). The problem of computing the high-dimensional failure probability integral (1) is then reduced to a number of conditional onedimensional problems, which are solved along the "important direction" $\underline{\alpha}$ in the standard normal space. The conditional one-dimensional failure probabilities (associated to the conditional one-dimensional problems) are readily computed by using the standard normal cumulative distribution function (Pradlwarter et al. 2005).
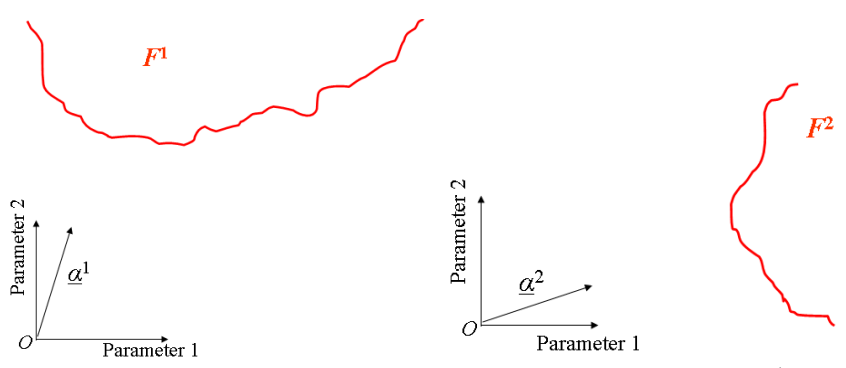

Figure 2. Examples of possible important unit vectors $\underline{\alpha}^{1}$ (left) and $\underline{\alpha}^{2}$ (right) pointing towards the corresponding failure domains $F^{1}$ (left) and $F^{2}$ (right) in a two-dimensional uncertain parameter space.

\subsection{Transformation of the physical space into the standard normal space}

Let $\underline{x}=\left\{x_{1}, x_{2}, \ldots, x_{j}, \ldots, x_{n}\right\} \in \Re^{n}$ be the vector of uncertain parameters defined in the original physical space $\underline{x} \in \Re^{n}$. For problems where the dimension $n$ is not so small, the parameter vector $\underline{x}$ can be transformed into the vector $\underline{\theta} \in \mathfrak{R}^{n}$, where each element of the vector $\theta_{j}, j=1,2, \ldots, n$, is associated with a central unit Gaussian standard distribution (Schueller et al. 2004). The joint probability density function of the random parameters $\left\{\theta_{j}: j=1,2, \ldots, n\right\}$ is, then, $\varphi(\theta)=\Pi_{j=1}^{n} \phi_{j}\left(\theta_{j}\right)$, where $\phi_{j}\left(\theta_{j}\right)=1 / \sqrt{2 \pi} \cdot \exp (-$ $\left.\theta_{j}^{2} / 2\right), j=1,2, \ldots, n$.

The mapping from the original, physical vector of random variables $x \in \mathfrak{R}^{n}$ to the standard normal vector $\underline{\theta} \in \mathfrak{R}^{n}$ is denoted by $T_{x \theta}(\cdot)$ and its inverse by $T_{\theta x}(\cdot)$, i.e., $\underline{\theta}=T_{x \theta}(\underline{x})$ and $\underline{x}=T_{\theta x}(\underline{\theta})$, respectively.

These transformations are in general nonlinear and are obtained by applying Rosenblatt's or Nataf's transformations, respectively (Huang \& Du 2006). They are linear only if the random vector $x$ is jointly Gaussian distributed. By transformation $\underline{\theta}=T_{x \theta}(\underline{x})$, also the Performance Function (PF) or Limit State Function (LSF) $g_{x}(\cdot)$ defined in the physical space (Section 1) can be transformed into $g_{\theta}(\cdot)$ in the standard normal space:

$g_{\theta}(\underline{\theta})=g_{x}(\underline{x})=g_{x}\left(T_{\theta x}(\underline{\theta})\right)$

Since in most cases of practical interest the function $g_{\theta}(\cdot)$ is not known analytically, it can be evaluated only point-wise. According to (3), the evaluation of the system performance function $g_{\theta}(\cdot)$ at a given point $\underline{\theta}^{k}, k=1,2, \ldots, N_{T}$, in the standard normal space requires i) a transformation into the original space, ii) a complete simulation of the system response and iii) the computation of the system response from the model. The computational cost of evaluating the failure probability is governed by the number of system performance analyses that have to be carried out (Schueller et al. 2004).

\subsection{The important direction $\underline{\alpha}$ for Line Sampling}

Three methods have been proposed to estimate the important direction $\alpha$ for Line Sampling. In (Koutsourelakis et al. 2004), the important unit vector $\underline{\alpha}$ is taken as pointing in the direction of the "design point" in the standard normal space. According to a geometrical interpretation, the "design point" is defined as the vector point $\underline{\theta}^{*}$ on the limit state surface $g_{\theta}(\underline{\theta})$ which is closest to the origin in the standard normal space. Then, the unit important vector $\underline{\alpha}$ can be easily obtained by normalizing $\underline{\theta}^{*}$, i.e., $\alpha=$ $\underline{\theta}^{*} /\left\|\underline{\theta}^{*}\right\|_{2}$, where $\|\cdot\|_{2}$ denotes the usual Euclidean measure of a vector.

In (Pradlwarter et al. 2005), the direction of $\underline{\alpha}$ is identified as the normalized gradient of the performance function in the standard normal space. Finally, the important unit vector $\underline{\alpha}$ can also be computed as the normalized "center of mass" of the failure domain $F$ of interest (Koutsourelakis et al. 2004). A point $\underline{\theta}^{0}$ is taken in the failure domain $F$. This can be done by traditional Monte Carlo sampling or by engineering judgment when possible. Subsequently, $\underline{\theta}^{0}$ is used as the initial point of a Markov chain which lies entirely in the failure domain $F$. For that purpose a MCMC Metropolis-Hastings algorithm is employed to generate a sequence of $N_{s}$ points $\left\{\underline{\theta}^{u}: u=\right.$ $\left.1,2, \ldots, N_{s}\right\}$ lying in the failure domain $F$ (Metropolis et al. 1956, Au \& Beck 2003): in extreme synthesis, a candidate point $\widetilde{\theta}^{u+1}$ is generated from the current point $\underline{\theta}^{u}$ by random sampling from a userdefined 'proposal' probability distribution; then, either the candidate point $\widetilde{\widetilde{\theta}}^{u+1}$ or the current point $\underline{\theta}^{u}$ is taken as the next point of the Markov chain, depending on whether the candidate $\widetilde{\theta}^{u+1}$ lies in the failure region $F$ or not. The unit vectors $\underline{\theta}^{u} /\left\|\underline{\theta}^{u}\right\|_{2}, u=$ $1,2, \ldots, N_{s}$, are then averaged in order to obtain the LS important unit vector as $\underline{\alpha}=1 / N_{s} \cdot \sum_{u=1}^{N_{s}} \underline{\theta}^{u} /\left\|\underline{\theta}^{u}\right\|_{2}$ (Figure 3). This direction is by no means optimal, but it is clear that it provides a good approximation of the important regions of the failure domain (at least as the sample size $N_{s}$ is large). On the other hand, it should be noticed that the procedure implies $N_{s}$ additional system analyses by the deterministic model simulating the system, which substantially increase the computational cost associated to the simulation method.

In the implementation of LS for this work, the method based on the normalized "center of mass" of the failure domain $F$ has been employed, because it relies on a "map" approximating the failure domain 
$F$ under analysis (given by the failure samples generated through a Markov chain) and thus it provides in principle the most realistic and reliable estimate for the LS important direction $\underline{\alpha}$.

For completeness, a thorough description of the Line Sampling algorithm and its practical implementation issues is given in the Appendix at the end of the paper.

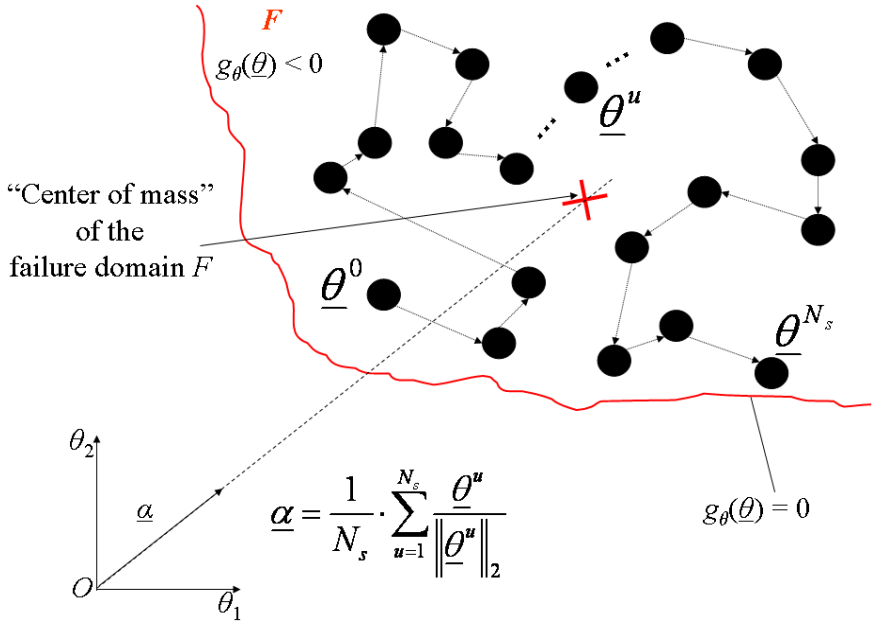

Figure 3. Line Sampling important unit vector $\underline{\alpha}$ taken as the normalized "center of mass" of the failure domain $F$ in the standard normal space. The "center of mass" of $F$ is computed as an average of $N_{s}$ failure points generated by means of a Markov chain starting from an initial failure point $\underline{\theta}^{0}$ (Koutsourelakis et al. 2004).

\section{APPLICATION: THE CRACKED PLATE MODEL}

The cracked plate model is a classical example in Fracture Mechanics and its relative simplicity allows a detailed and complete study of different simulation techniques. A thorough description of this model can be found in (Ardillon \& Venturini 1995).

\subsection{The mechanical model}

A metal plate of infinite length with a defect of initial length equal to $a[\mathrm{~m}]$ is considered. The plate is supposed to be subject to a uniform normal loading (i.e., stress) $s_{\infty}[\mathrm{MPa}]$. The intensity factor $K$ $[\mathrm{MPa} \sqrt{\mathrm{m}}]$, determined by the uniform loading in the neighborhood of the defect is defined as follows:

$$
K=F s_{\infty} \sqrt{\pi a}
$$

where $F$ is the shape factor of the defect. The plate is supposed to break (i.e., fail) when the intensity factor $K$ in (4) becomes greater than or equal to a critical value $K_{c}$, i.e.:

$$
K=F S_{\infty} \sqrt{\pi a} \geq K_{c}
$$

\subsection{The structural reliability model}

From the point of view of a structural reliability analysis, the cracked plate mechanical model of Section 4.1 is analyzed within a probabilistic framework in which the variables $K_{c}, a, F$ and $s_{\infty}$ are uncertain (for simplicity of illustration with respect to the notation of the previous Sections, the four variables are hereafter named $x_{1}, x_{2}, x_{3}$ and $x_{4}$, respectively).

Referring to (5), the performance function $g_{x}(\underline{x})$ of the system is

$$
g_{x}(\underline{x})=g_{x}\left(x_{1}, x_{2}, x_{3}, x_{4}\right)=x_{1}-x_{3} x_{4} \sqrt{\pi x_{2}}
$$

The failure region $F$ is then expressed as

$$
F=\left\{\underline{x}: g_{x}(\underline{x}) \leq 0\right\}=\left\{\left(x_{1}, x_{2}, x_{3}, x_{4}\right): x_{1} \leq x_{3} x_{4} \sqrt{\pi x_{2}}\right\}(7)
$$

Finally, the probability of system failure $P(F)$ is written as follows:

$$
P(F)=P\left[g_{x}(\underline{x}) \leq 0\right]=P\left(x_{1} \leq x_{3} x_{4} \sqrt{\pi x_{2}}\right) \text {. }
$$

\subsection{Case studies}

Four case studies, namely Case 0 (Reference case), 1,2 and 3, are considered with respect to the structural reliability model of the previous Section 4.2. Each case study is characterized by different PDFs for the uncertain variables $x_{1}, x_{2}, x_{3}$ and $x_{4}$ and by different failure probabilities $P(F)$ : these features are summarized in Table 1 . It is worth noting that the exact (i.e., analytically computed) failure probabilities $P(F)$ approximately range from $10^{-3}$ to $10^{-7}$, allowing a deep exploration of the capabilities of the simulation algorithms considered and a meaningful comparison between them (Gille 1999). Finally, notice that the uncertain variables in Table 1 are independent and most of the corresponding PDFs are Gaussian. Although this is one of the most favourable cases for the transformation of the physical space into the standard normal space, the application of Rosenblatt's or Nataf's transformations to possibly correlated, non-Gaussian variables would add only little computational burden to the method (Huang \& Du, 2006).

Table 1. Probability distributions and parameters (i.e., means and standard deviations) of the uncertain variables $x_{1}, x_{2}, x_{3}$, and $x_{4}$ of the cracked plate model of Section 4.2 for the four case studies considered (i.e., Cases 0, 1, 2 and 3); the last row reports the values of the corresponding exact (i.e., analytically computed) failure probabilities, $P(F)$ (Gille 1999).

\begin{tabular}{c|cccc}
\hline & Case 0 & Case 1 & Case 2 & Case 3 \\
\hline $\boldsymbol{x}_{\mathbf{1}}$ & $N(149.3,22.2)$ & $N(149.3,22.2)$ & $N(160,18)$ & $L G(149.3,22.2)$ \\
$\boldsymbol{x}_{\mathbf{2}}$ & $N\left(5 \cdot 10^{-3}, 10^{-3}\right)$ & $N\left(5 \cdot 10^{-3}, 10^{-3}\right)$ & $N\left(5 \cdot 10^{-3}, 10^{-3}\right)$ & $L G\left(5 \cdot 10^{-3}, 10^{-3}\right)$ \\
$\boldsymbol{x}_{\mathbf{3}}$ & $N(0.99,0.01)$ & $N(0.99,0.01)$ & $N(0.99,0.01)$ & $L G(0.99,0.01)$ \\
$\boldsymbol{x}_{\mathbf{4}}$ & $N(600,60)$ & $N(300,30)$ & $N(500,45)$ & $L G(600,60)$ \\
$\boldsymbol{P (} \boldsymbol{F})$ & $1.165 \cdot 10^{-3}$ & $4.500 \cdot 10^{-7}$ & $4.400 \cdot 10^{-7}$ & $3.067 \cdot 10^{-4}$ \\
\hline
\end{tabular}




\subsection{Results}

In this Section, the results of the application of SS and LS for the reliability analysis of the cracked plate model of Section 4.1 are illustrated with reference to Case studies 0, 1, 2 and 3 described in the previous Section 4.3.

For fair comparison, all methods have been run with a total of $N_{T}=50000$ samples in all four cases. The efficiency of the simulation methods under analysis is evaluated in terms of four quantities: the failure probability estimate $\hat{P}(F)$, the sample standard deviation $\hat{\sigma}$ of the failure probability estimate $\hat{P}(F)$, the coefficient of variation (c.o.v.) $\delta$ of $\hat{P}(F)$ (defined as the ratio of the sample standard deviation $\hat{\sigma}$ to the estimate $\hat{P}(F)$ ) and the Figure Of Merit (FOM) of the method (defined as $1 /\left(\hat{\sigma}^{2} \cdot t_{\text {comp }}\right)$, where $t_{\text {comp }}$ is the computational time required by the simulation method). The closer is the estimate $P(F)$ to the exact (i.e., analytically computed) failure probability $P(F)$, the more accurate is the simulation method. However, since $\hat{P}(F)$ is a statistical estimation of the exact result, it could happen that a less accurate result leads to an estimate closer to the exact solution; thus, the estimate $\hat{P}(F)$ has to be combined to the standard deviation (and/or other quality indicators) to play this role. The sample standard deviation $\hat{\sigma}$ and the c.o.v. $\delta$ of $\hat{P}(F)$ are used to quantify the variability of the failure probability estimator; in particular, the lower are the values of $\hat{\sigma}$ and $\delta$, the lower is the variability of the corresponding failure probability estimator and thus the higher is the efficiency of the simulation method adopted.

Finally, the FOM is introduced to take into account the computational time required by the method. The value of the FOM increases as the sample variance $\hat{\sigma}^{2}$ of the failure probability estimate $\hat{P}(F)$ and the computational time $t_{\text {comp }}$ required by the method decrease; thus, in this case the higher is the value of the index, the higher is the efficiency of the method (Rubinstein 1981).

The different simulation methods are also compared with respect to two direct performance indicators relative to standard MCS. First, the ratio of the sample standard deviation $\hat{\sigma}_{M C}$ obtained by Standard MCS to that obtained by the simulation method under analysis $\hat{\sigma}_{\text {meth }}$ is computed. This ratio only quantifies the improvement in the precision of the estimate achieved by using a given simulation method instead of standard MCS. Then, the ratio of the FOM of the simulation method in object, namely $\mathrm{FOM}_{\text {meth }}$, to that of standard MCS, namely $\mathrm{FOM}_{M C}$, is considered to quantify the overall improvement in efficiency achieved by a given simulation method with respect to standard MCS, since it takes into account also the computational time required. Obviously, the higher are the values of these two indices for a given method, the higher is the efficiency of that method (Gille 1999).
Table 2 reports the values of $\hat{P}(F), \hat{\sigma}, \delta$, FOM, $\hat{\sigma}_{M C} / \hat{\sigma}_{\text {meth }}$ and $\mathrm{FOM}_{\text {meth }} / \mathrm{FOM}_{M C}$ obtained by Standard MCS, SS and LS in Cases 0, 1, 2 and 3 (Section 4.3 ); the actual number $N_{\text {sys }}$ of system response analyses (i.e., model evaluations) is also reported. Notice that for both SS and LS the actual number $N_{s y s}$ of system analyses does not coincide with the total number $N_{T}$ of random samples drawn (i.e., $N_{T}=$ 50000). In particular, in the SS method, the presence of repeated conditional samples in each Markov chain (used to gradually populate the intermediate event regions) allows a reduction in the number of model evaluations required: actually, one evaluation is enough for all identical samples. In the LS method, instead, the actual number $N_{s y s}$ of system analyses is given by $N_{s y s}=N_{S}+2 \cdot N_{T}$ : in particular, $N_{s}=2000$ analyses are performed to generate the Markov chain used to compute the important unit vector $\underline{\alpha}$ as the normalized "center of mass" of the failure domain $F$ (Section 3.2); the $2 \cdot N_{T}$ analyses are carried out to compute the $N_{T}$ conditional onedimensional probability estimates $\left\{\hat{P}^{k}(F): k=1,2\right.$, $\left.\ldots, N_{T}\right\}$ by linear interpolation (equation (3') in the Appendix).

It can be seen that SS performs consistently better than standard MCS and its performance significantly grows as the failure probability to be estimated decreases: for instance, in Case 0 (Reference), where $P(F) \sim 10^{-3}$, the FOM of SS, namely $\mathrm{FOM}_{S S}$, is only four times larger than that of Standard MCS, namely $\mathrm{FOM}_{M C}$; whereas in Case 1 , where $P(F) \sim 10^{-7}$, the ratio $\mathrm{FOM}_{S S} / \mathrm{FOM}_{M C}$ is about 557.

On the other hand, LS outperforms SS with respect to both $\hat{\sigma}_{M C} / \hat{\sigma}_{\text {meth }}$ and $\mathrm{FOM}_{\text {meth }} / \mathrm{FOM}_{M C}$ in all the Cases considered. For instance, in Case 2, where the failure probability $P(F)$ to be estimated is very small, i.e., $P(F)=4.4 \cdot 10^{-7}$, the ratio $\hat{\sigma}_{M C} / \hat{\sigma}_{L S}$ is 155 times larger than the ratio $\hat{\sigma}_{M C} / \hat{\sigma}_{S S}$, whereas the ratio $\mathrm{FOM}_{L S} / \mathrm{FOM}_{M C}$ is 11750 times larger than the ratio $\mathrm{FOM}_{S S} / \mathrm{FOM}_{M C}$. Notice that for the LS method even though the determination of the sampling important direction $\underline{\alpha}$ (Section 3.2) and the calculations of the conditional one-dimensional failure probability estimates $\left\{\hat{P}^{k}(F): k=1,2, \ldots, N_{T}\right\}$ (equation (3') in the Appendix) require much more than $N_{T}$ system analyses by the model, this is significantly overweighed by the accelerated convergence rate that can be attained by the LS method with respect to SS.

Finally, it is also worth noting that the use of preferential lines (instead of random points) to probe the failure domain $F$ of interest makes the effectiveness of the LS method almost independent of the target failure probability $P(F)$ to be estimated: for example, the value of the c.o.v. $\delta$ is almost the same for values of the target failure probability $P(F)$ which change by four orders of magnitude (in particular, $\delta=$ $4.399 \cdot 10^{-4}, 3.986 \cdot 10^{-4}, 1.015 \cdot 10^{-3}$ and $5.923 \cdot 10^{-4}$ for $P(F) \sim 1.169 \cdot 10^{-3}, 4.493 \cdot 10^{-7}, 4.381 \cdot 10^{-7}$ and $3.068 \cdot 10^{-4}$, respectively). 
Table 2. Results of the application of standard MCS, SS and LS to the reliability analysis of Cases 0 (Reference), 1, 2 and 3 of the cracked plate model of Section 4.2; the values of the performance indicators used to compare the effectiveness of the methods (i.e., $\hat{\sigma}_{M C} / \hat{\sigma}_{m e t h}$ and $\mathrm{FOM}_{\text {meth }} / \mathrm{FOM}_{M C}$ ) are highlighted in bold.

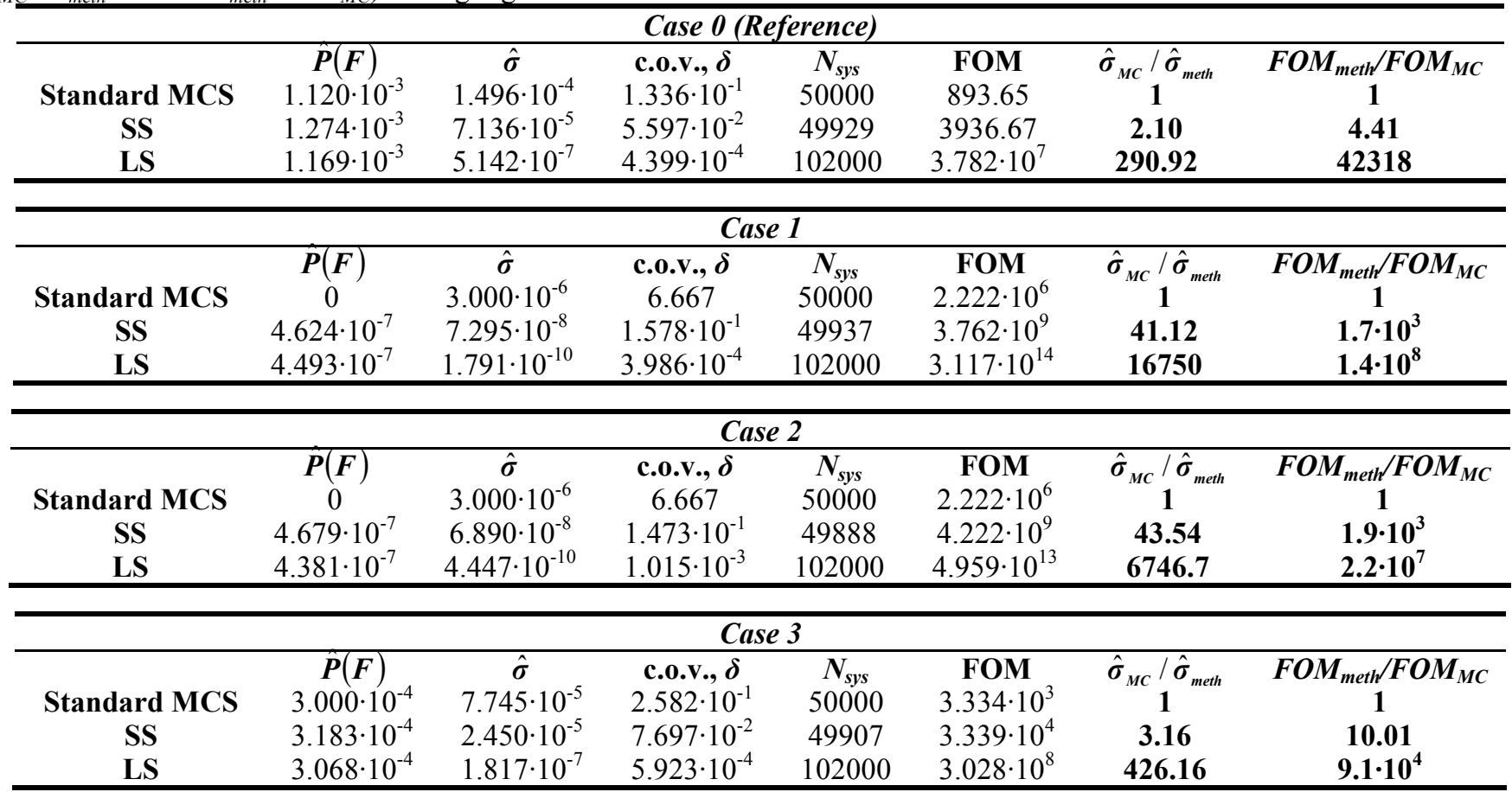

\section{CONCLUSIONS}

One of the major obstacles in applying simulation methods for the reliability analysis of engineered systems and structures is the challenge posed by the estimation of small failure probabilities: the simulation of the rare events of failure occurrence implies a significant computational burden (Schueller 2007).

In this paper, the Subset Simulation (SS) and Line Sampling (LS) methods have been considered for improving the efficiency of Monte Carlo Simulation (MCS) in the estimation of system failure probability. A structural reliability model of literature, i.e. the cracked plate model, has been taken as benchmark to test the two methods.

The results of SS and LS have been compared to those of standard MCS in the estimation of failure probabilities as small as $10^{-7}$. The results have demonstrated that SS becomes more and more efficient over standard MCS as the target probability of failure gets smaller. On the other hand, LS has been found to significantly outperform SS, in particular in the task of estimating very small failure probabilities (i.e., around $10^{-7}$ ).

Further, it has been shown that the use of preferential lines (instead of random points) to probe the failure domain of interest makes the effectiveness of the LS methodology almost independent of the failure probability to be estimated: this renders LS the most suitable method for an extremely wide range of real-world reliability problems.

\section{REFERENCES}

Ardillon, E. \& Venturini, V. 1995. Measures de sensibilitè dans les approaches probabilistes. Rapport EDF HP16/95/018/A.

Au, S. K. \& Beck, J. L. 2001. Estimation of small failure probabilities in high dimensions by subset simulation. Probabilist. Eng. Mech. 16(4): 263-277.

Au, S. K. \& Beck, J. L. 2003. Subset Simulation and its application to seismic risk based on dynamic analysis. J. Eng. Mech.-ASCE 129(8): 1-17.

Gille, A. 1999. Probabilistic numerical methods used in the applications of the structural reliability domain. PhD Thesis, Universitè Paris 6.

Huang, B. \& Du, X. 2006. A robust design method using variable transformation and Gauss-Hermite integration. Int. J. Numer. Methods Eng. 66: 1841-1858.

Koutsourelakis, P. S., Pradlwarter, H. J., Schueller, G. I. 2004. Reliability of structures in high dimensions, Part I: algorithms and application. Probab. Eng. Mech. 19: 409-417.

Metropolis, N., Rosenbluth, A. W., Rosenbluth, M. N. \& Taller, A. H. 1953. Equations of state calculations by fast computing machines. J. Chem. Phys. 21(6): 1087-1092.

Nutt, W. T. \& Wallis, G. B. 2004. Evaluations of nuclear safety from the outputs of computer codes in the presence of uncertainties. Reliab. Eng. Syst. Safe. 83: 57-77.

Patalano, G., Apostolakis, G. E., Hejzlar, P. 2008. Risk informed design changes in a passive decay heat removal system. Nucl. Technol. 163: 191-208.

Pradlwarter, H. J., Pellissetti, M. F., Schenk, C. A., Schueller, G. I., Kreis, A., Fransen, S., Calvi, A. \& Klein, M. 2005. Realistic and efficient reliability estimation for aerospace structures. Comput. Meth. Appl. Mech. Eng. 194: 15971617.

Rubinstein, R. Y. 1981. Simulation and the Monte Carlo method, Wiley.

Schueller, G. I. 2007. On the treatment of uncertainties in structural mechanics and analysis. Comput. Struct. 85: 235-243. 
Schueller, G. I., Pradlwarter, H. J. \& Koutsourelakis, P. S. 2004. A critical appraisal of reliability estimation procedures for high dimensions. Probab. Eng. Mech., 19: 463474.

Schueller, G. I. \& Pradlwarter, H. J. 2007. Benchmark study on reliability estimation in higher dimension of structural systems - An overview. Struct. Saf. 29: 167-182.

\section{APPENDIX: THE LINE SAMPLING ALGORITHM}

The LS algorithm proceeds as follows (Pradlwarter et al., 2005):

1 Determine the unit important direction $\underline{\alpha}=\left\{\alpha_{1}\right.$, $\left.\alpha_{2}, \ldots, \alpha_{j}, \ldots, \alpha_{n}\right\}$. Any of the methods summarized in Section 3.2 can be employed to this purpose. Notice that the computation of $\underline{\alpha}$ implies additional system analyses, which substantially increase the computational cost associated to the simulation method (Section 3.2).

2 From the original multidimensional joint probability density function $q(\cdot): \mathfrak{R}^{n} \rightarrow[0, \infty)$, sample $N_{T}$ vectors $\left\{\underline{x}^{k}: k=1,2, \ldots, N_{T}\right\}$, with $\underline{x}^{k}=\left\{x_{1}{ }^{k}\right.$, $\left.x_{2}{ }^{k}, \ldots, x_{j}^{k}, \ldots, x_{n}{ }^{k}\right\}$ by standard MCS.

3 Transform the $N_{T}$ sample vectors $\left\{\underline{x}^{k}: k=1,2, \ldots\right.$, $\left.N_{T}\right\}$ defined in the original (i.e., physical) space of possibly dependent, non-normal random variables (step 2. above) into $N_{T}$ samples $\left\{\underline{\theta}^{k}: k=1,2, \ldots\right.$, $\left.N_{T}\right\}$ defined in the standard normal space where each component of the vector $\underline{\theta}^{k}=\left\{\theta_{1}{ }^{k}, \theta_{2}{ }^{k}, \ldots\right.$, $\left.\theta_{j}^{k}, \ldots, \theta_{n}{ }^{k}\right\}, k=1,2, \ldots, N_{T}$, is associated with an independent central unit Gaussian standard distribution (Section 3.1).

4 Estimate $N_{T}$ conditional "one-dimensional" failure probabilities $\left\{\hat{P}^{k}(F): k=1,2, \ldots, N_{T}\right\}$, corresponding to each one of the standard normal samples $\left\{\underline{\theta}^{k}: k=1,2, \ldots, N_{T}\right\}$ obtained in step 3 . above. In particular, for each random sample $\underline{\theta}^{k}, k$ $=1,2, \ldots, N_{T}$, perform the following steps (Figure 1') (Schueller et al. 2004, Pradlwarter et al. 2005):

a. Define the sample vector $\underline{\widetilde{\theta}}^{k}, k=1,2, \ldots, N_{T}$, as the sum of a deterministic multiple of $\underline{\alpha}$ and a vector $\underline{\theta}^{k, \perp}, k=1,2, \ldots, N_{T}$, perpendicular to the direction $\underline{\alpha}$, i.e.,

$\underline{\tilde{\theta}}^{k}=c^{k} \underline{\alpha}+\underline{\theta}^{k, \perp}, k=1,2, \ldots, N_{T}$

where $c^{k}$ is a real number in $[-\infty,+\infty]$ and

$$
\underline{\theta}^{k, \perp}=\underline{\theta}^{k}-\left\langle\underline{\alpha}, \underline{\theta}^{k}\right\rangle \underline{\alpha}, k=1,2, \ldots, N_{T}
$$

In (2'), $\underline{\theta}^{k}, k=1,2, \ldots, N_{T}$, denotes a random realization of the input variables in the standard normal space of dimension $n$ and $\langle\underline{\alpha}$, $\left.\underline{\theta}^{k}\right\rangle$ is the scalar product between $\underline{\alpha}$ and $\underline{\theta}^{k}, k=$ $1,2, \ldots, N_{T}$. Finally, it is worth noting that since the standard Gaussian space is iso- tropic, both the scalar $c^{k}$ and the vector $\underline{\theta}^{k, \perp}$ are also standard normally distributed (Pradlwarter et al. 2005).

b. Compute the value $\bar{c}^{k}$ as the intersection between the limit state function $g_{\theta}\left(\widetilde{\theta}^{k}\right)=g_{\theta}\left(c^{k} \underline{\alpha}\right.$ $\left.+\underline{\theta}^{k, \perp}\right)=0$ and the line $l^{k}\left(c^{k}, \underline{\alpha}\right)$ passing through $\underline{\theta}^{k}$ and parallel to $\underline{\alpha}$ (Figure 1'). The value of $\bar{c}^{k}$ can be approximated by evaluating the performance function $g_{\theta}(\cdot)$ at two or three different values of $c^{k}$ (e.g., $c_{1}{ }^{k}, c_{2}{ }^{k}$ and $c_{3}{ }^{k}$ in Figure 1'), fitting a first or second order polynomial and determining its root (Figure 1').

c. Solve the conditional one-dimensional reliability problem associated to each random sample $\underline{\theta}^{k}, k=1,2, \ldots, N_{T}$, in which the only (standard normal) random variable is $c^{k}$. The associated conditional failure probability $\hat{P}^{k}(F), k=1,2, \ldots, N_{T}$, is given by

$$
\begin{aligned}
\hat{P}^{k}(F)= & P\left[N(0,1)>\bar{c}^{k}\right]= \\
& 1-P\left[N(0,1) \leq \bar{c}^{k}\right]= \\
& 1-\Phi\left(\bar{c}^{k}\right)=\Phi\left(-\bar{c}^{k}\right)
\end{aligned}
$$

where $\Phi(\cdot)$ denotes the standard normal cumulative distribution function.

5 Using the independent conditional "onedimensional" failure probability estimates $\left\{\hat{P}^{k}(F): k=1,2, \ldots, N_{T}\right\}$ in (3') (step 4.c. above), compute the unbiased estimator $\hat{P}^{k}(F)$ for the failure probability $P(F)$ as

$$
\hat{P}(F)=\frac{1}{N_{T}} \sum_{k=1}^{N_{T}} \hat{P}^{k}(F)
$$

The variance of the estimator (4') is

$$
\sigma^{2}(\hat{P}(F))=\frac{1}{N_{T}\left(N_{T}-1\right)} \sum_{k=1}^{N_{T}}\left(\hat{P}^{k}(F)-\hat{P}(F)\right)^{2}
$$

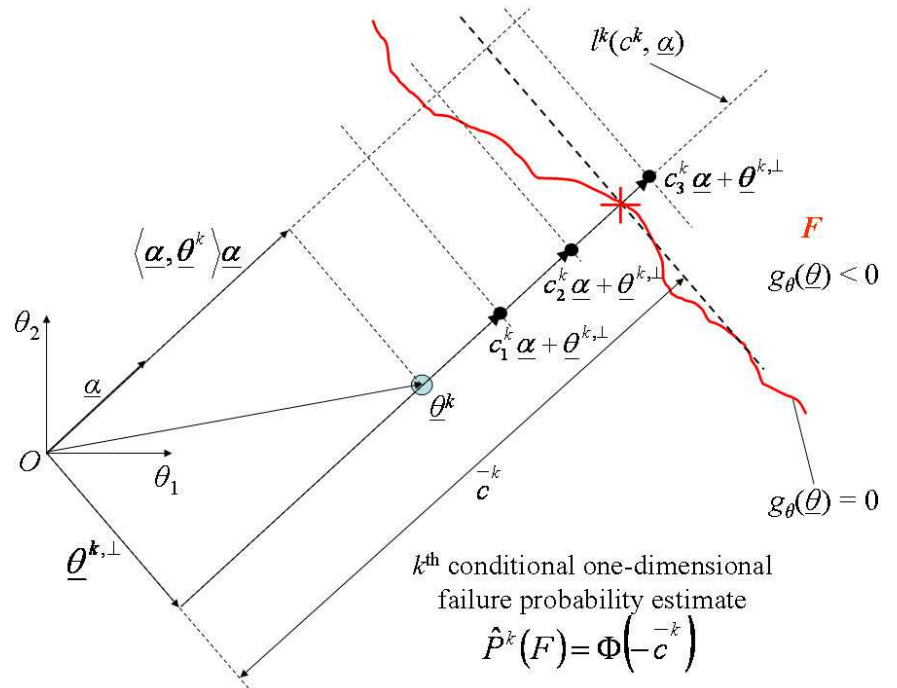

Figure 1'. The Line Sampling procedure (Pradlwarter et al. 2005) 